\title{
The effect of marker variability on the discrimination of temporal intervals
}

\author{
M. J. PENNER \\ Hunter College of the City University of New York, New York, New York 10021
}

\begin{abstract}
This research investigates the human observer's ability to discriminate between the durations of two silent intervals, each interval preceded and followed by noise bursts called markers. The markers are separated by $T$ or $T+\Delta T^{\prime} m s e c$ and $T$ ranges from 0.3 to $1,000 \mathrm{msec}$. $\Delta T$ is defined as that value of $\Delta T^{\prime}$ for which the probability of discriminating $T$ from $T+\Delta T^{\prime}$ is 0.75 . We compared the value of $\Delta T$ for conditions in which the markers were fixed in amplitude and duration with conditions in which the marker amplitudes and durations were randomly chosen. $\Delta T$ increased by as much as a factor of 4 when the amplitude and duration of the markers were randomized. The performance decrement was primarily due to randomizing the first marker duration.
\end{abstract}

Models of duration discrimination relate discriminability to the duration of the stimuli presented. Stimuli that differ in duration, however, differ in other ways as well, and the idea that it is only their temporal extent which provides a discrimination cue is open to question. In a recent paper, Abel (1972) investigated the human observer's ability to discriminate between the durations of two silent intervals, each interval preceded and followed by noise bursts called markers. This paradigm is an important one, because the silent intervals differ in temporal extent only. There are no cues related to the total energy or to the energy spectrum of the stimuli. It is therefore of particular interest whether or not discriminability is independent of marker amplitude and duration in this paradigm.

In Abel's paradigm, the marker amplitude and duration were fixed from trial to trial and varied between blocks. For these fixed markers, discrimination was affected by marker amplitude indicating that some nontemporal cue might be used (Allan \& Kristofferson, 1974). In the present experiment, we investigate an extreme case of marker variability. In our work, the parameters of each of the four markers bounding the two intervals are chosen randomly and independently. For large marker variability, we reopen the question of whether it is only the physical duration of the silent temporal interval which provides the discrimination cue.

\section{METHODS}

Subjects

Two undergraduates at Arizona State University were paid to participate. The author was also a subject. Two of the three

This research was supported in part by a grant from the National Institutes of Health and in part by a faculty research award from the City University of New York. subjects were practiced listeners with at least 3 years of experience in psychoacoustic experiments.

\section{Apparatus}

The durations of the noises and the gap between them were controlled by a PDP-15 computer. The noise was generated by a random noise generator (General Radio Model 1383), gated, filtered ( 0 to $20 \mathrm{kHz}$; Krohnhite Model 3550R), and then served as input to TDH-39 headphones. Two observers were run simultaneously in two double-walled IAC chambers.

\section{Procedure}

The data were collected using a two-interval forced-choice procedure. A trial began with a flash of a warning light. Two silent intervals, a standard interval, or base ( $\mathrm{T} \mathrm{msec}$ ) and a comparison interval $\left(T+\Delta T^{\prime}\right.$ msec) were then presented. The silent intervals were preceded and followed by noise bursts. The standard gap, $T$, was equally likely to occur in the first or the second interval on each trial. The time between the offset of the second marker for the first interval and the onset of the first marker for the second interval was fixed at $1 \mathrm{sec}$. The observer selected the interval he thought to have contained the shorter gap. At the end of a trial, a flash of light indicated the correct choice, which was arbitrarily designated as the standard. An adaptive procedure which tracked the $75 \%$ correct point in a 2IFC paradigm was employed. Six blocks of 50 trials each were used as the final estimate of threshold, so that there are 300 observations per subject per point. For Conditions 1-5 (see below), $\mathrm{T}$ ranged from $300 \mu \mathrm{sec}$ to $1 \mathrm{sec}$ in nine steps. For Condition 6, $T$ was fixed at $100 \mathrm{msec}$.

\section{Conditions}

An outline of the conditions is presented in Table 1. For the randomized situations, on each trial, the parameters of each of the markers bounding the two intervals were chosen randomly and independently (as described below). A control condition used fixed marker amplitude and duration, replicating Abel (1972). For conditions in which the marker amplitude was randomized, $N_{0}$, the noise power per cycle per second, ranged uniformly from 22 to $43 \mathrm{~dB}$ SPL in 3-dB steps. For conditions in which the marker duration was randomized, its duration ranged uniformly from 1 to $300 \mathrm{msec}$ in $1-\mathrm{msec}$ steps. For fixed marker conditions, the marker duration was usually $100 \mathrm{msec}$ with $\mathrm{N}_{\mathrm{o}}$ equal to $43 \mathrm{~dB}$ SPL except for Condition 5 , the dichotic condition, which utilized 10 -msec markers, and Condition $4 a$, a binaural control, which also utilized 10-msec markers. 
Table 1

Stimulus Conditions

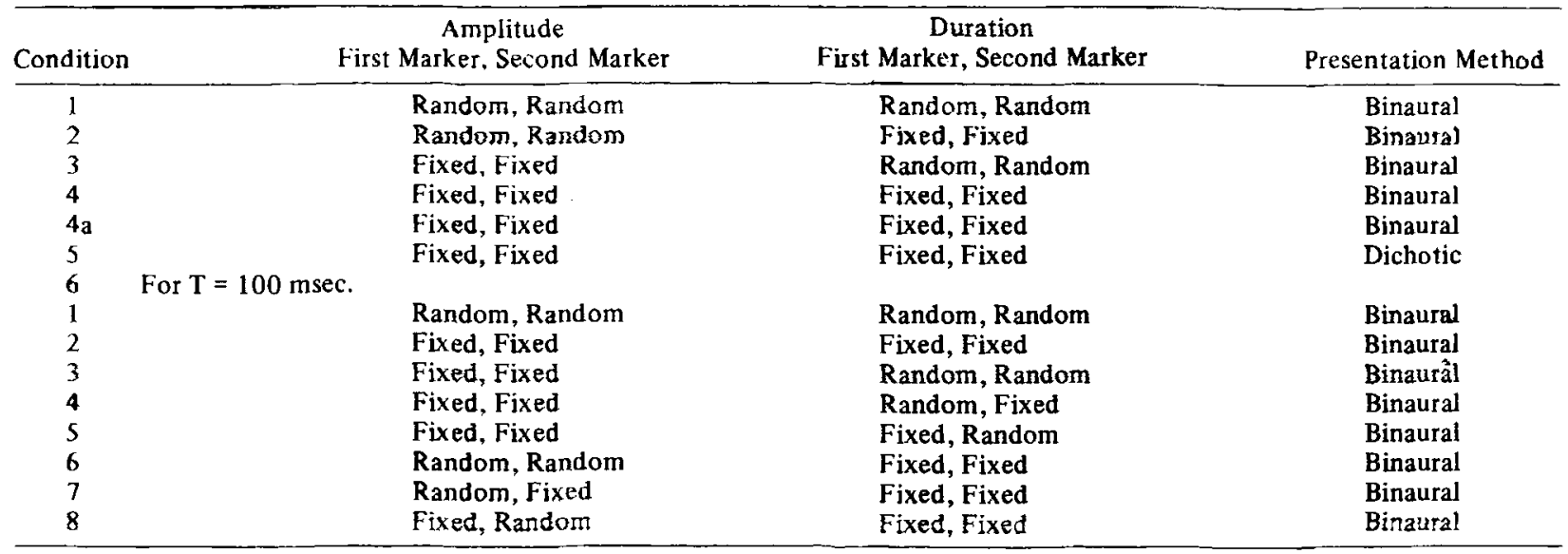

All conditions were run in blocks. That is, $T$ was fixed and $\Delta \mathrm{T}$ for the fixed $\mathrm{T}$ was determined. Each block of trials began with a comparison gap $(T+\Delta T)$ at a large value. The value of $\Delta T$ was then decreased using an adaptive procedure until the value of the comparison gap correctly discriminated from the standard on $75 \%$ of the trials was determined.

Before collecting any of the data reported here, the subjects practiced Condition 4 extensively. In order to insure that performance had asymptoted, each subject was run in Condition 4 until there was no longer a systematiz decrease over blocks in that value of the comparison gap, $\Delta T$, correctly discriminated from the standard on $75 \%$ of the trials. The practice ranged from $81020 \mathrm{~h}$.

Once performance had asymptoted, a condition was randomly selected. On a given day, the subjects ran for all values of $T$ in the condition selected for that day. For each condition, sufficient practice was allowed to insure that there was no systematic decrease over blocks in the value of $\Delta T$. After completing Conditions 1-5, Condition 6 was run. Finally, to check for long-term practice effects. all conditions were rerun with $\Delta T$ fixed at the subject's threshold. The data were quite consistent, and furthermore there was such small intersubject variability that the results for alt three subjects were averaged together.

\section{RESULTS}

Figure 1 displays the results from Conditions 1 through 5 (see Table 1), and the raw data are presented in Table 2. Both axes in Figure 1 are logarithmic, and $\Delta T$ is presented as a function of $T$. The estimate of $\Delta T$ is the value of $\Delta T^{\prime}$ for which the probability of discriminating $T$ from $T+\Delta T^{\prime}$ is 0.75 .

The data for Condition 4 (the $\times s$ ) in Figure 1 can be compared with two results in the literature. First, for Condition 4, our experiment replicates Abel's (1972). However, our values of $\Delta T$ are consistently smaller (i.e., our observers are more sensitive) than Abel's for T $>10 \mathrm{msec}$ (as are Divenyi \& Danner's, 1975, and Woodrow's, 1935). Second, for small values of $T$ in Condition 4 , the paradigm nearly reduces to Plomp's (1964).. In Plomp's paradigm, the subject is asked to discriminate a 400 -msec burst of noise and two 200 -msec bursts separated in time by $\Delta \mathrm{T} \mathrm{msec}$. In one of the condi- tions presented here, the noises are separated by a $T$ of $.300 \mathrm{msec}$, but this $\mathrm{T}$ is so small that the condition very nearly replicates Plornp's. From our Condition 4 data, for a $T$ of $.300 \mathrm{msec}$, a $\Delta T$ of about $1.9 \mathrm{msec}$ is obtained. For an equivalent $\mathrm{N}_{0}$ in Plomp's experiment, a $\Delta \mathrm{T}$ of about $2.6 \mathrm{msec}$ was obtained so that our results are in near agreement with Plomp's. Thus our results from Condition 4 nearly replicate those of two previous studies.

There are several noteworthy points about the results from the other conditions in Figure 1. First, if the markers are randomized in duration (from

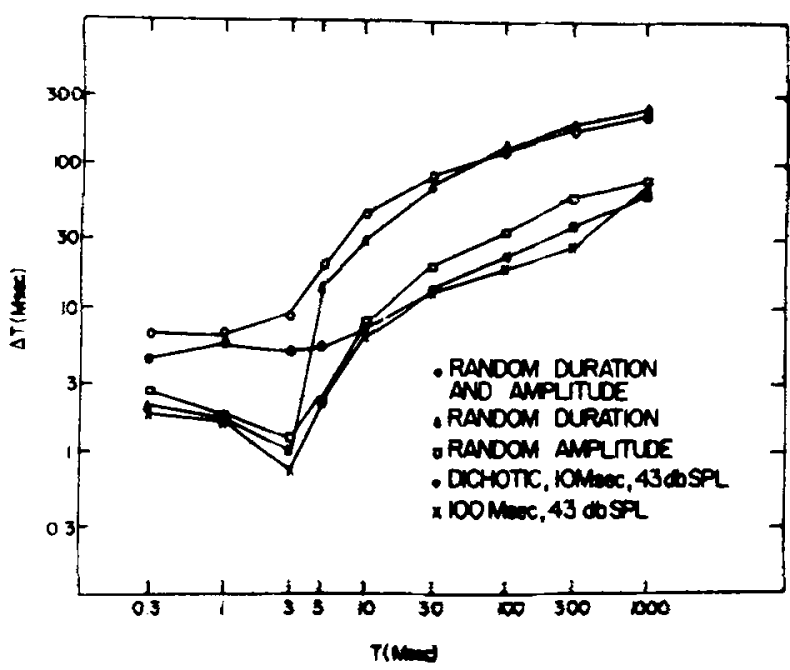

Figure 1. The just discriminable difference in gap duration as a function of the base duration. Three of the symbols represent the various randomized marker conditions as follows: open circles-marker durations randomized from 1 to 300 msec, marker amplitude randomized from $N_{0}=43$ to $N_{0}=23 \mathrm{~dB}$ SPL; triangles-marker duration randomized, amplitude at $\mathbf{N}_{\mathbf{0}}=$ $43 \mathrm{~dB}$ SPL; squares-marker amplitude randomized, marker duration fixed at 100 msec. For comparative purposes, two fixed marker conditions are also included. These are represented by the dots-a 10 -msec dichotic marker condilion with $N_{b}=43 \mathrm{~dB}$ SPL-and the $\times s$-the 100 -msec bingural marker condition with $N_{0}=43 \mathrm{~dB} \mathrm{SPL}$. 
1 to $300 \mathrm{msec}$ ) and in amplitude (over a $21-\mathrm{dB}$ range), performance is consistently worse than for the fixed marker conditions, even for $T$ as long as $1 \mathrm{sec}$. Second, the large shift in $\Delta T$ for $T>5$ msec seems to be primarily due to the random duration of the markers (for $T>5 \mathrm{msec}$, the triangles and the circles nearly overlap). The randomization of the amplitude of the markers has much less of an effect on $\Delta \mathrm{T}$ compared with the fixed conditions. Finally, note that, for $T>10 \mathrm{msec}$, the dichotic results are essentially the same as those in which the amplitude and duration of the markers are fixed and presented binaurally. However, for $T<10 \mathrm{msec}$, the dichotic and binaural conditions differ considerably. Presumably, peripheral cues are not available when the markers are presented dichotically. Therefore, our findings indicate that, for $\mathrm{T}<10 \mathrm{msec}$, judgments are based on peripheral cues (as Plomp, 1964, argues) rather than on the output of a central timing mechanism. Plomp suggested that the peripheral cue is the subjective intensity level at the onset of the second burst. This intensity difference is presumably due to peripheral interaction in the auditory system.

Comparing the $\times \mathrm{s}$ and the circles in Figure 1, it can be seen that $\Delta T$ changed dramatically when the durations of the markers were randomized. A logical question, then, concerns specification of the conditions that cause the change: is it the randomization of the first burst, the second burst, or both bursts that causes the increment in $\Delta T$ ? Figure 2 displays relevant results for the conditions in which $T$ was fixed at $100 \mathrm{msec}$ (Condition 6 in Table 1). The ordinate is again $\Delta \mathrm{T}$, but the abscissa now specifies the condition randomized. As can be seen, randomizing the duration of the first burst (Part 4 of Condition 6) affects discrimination nearly as much as randomizing the duration of both bursts (Part 3 of Condition 6). Randomizing the duration of the second burst (Part 5 of Condition 6) also affects discrimination, but less so than randomization of the first. Conditions in which only the amplitudes are randomized (Parts 6, 7, and 8 of Condition 6)

Table 2

Conditions

\begin{tabular}{lcccccc}
\hline T (msec) & 1 & 2 & 3 & 4 & $4 \mathrm{a}$ & 5 \\
\hline 0.3 & 6.7 & 2.7 & 2.1 & 1.8 & 2.0 & 4.3 \\
1 & 6.3 & 1.9 & 1.7 & 1.7 & 1.7 & 5.5 \\
3 & 9 & 1.3 & 1 & 0.8 & 0.6 & 5 \\
5 & 20 & 2.3 & 14 & 2.3 & 3. & 5.4 \\
10 & 45 & 8.7 & 30 & 7.5 & 8.7 & 7.3 \\
30 & 86 & 20 & 70 & 14.3 & 15 & 14 \\
100 & 125 & 35 & 131 & 19.7 & 23 & 24 \\
300 & 165 & 59 & 190 & 25.3 & 29 & 38 \\
1000 & 214 & 70 & 214 & 70 & 70 & 65 \\
\hline
\end{tabular}

Note - Values of $\Delta T$, for given $T$, in Conditions 1 through 5 . Entries are in milliseconds.

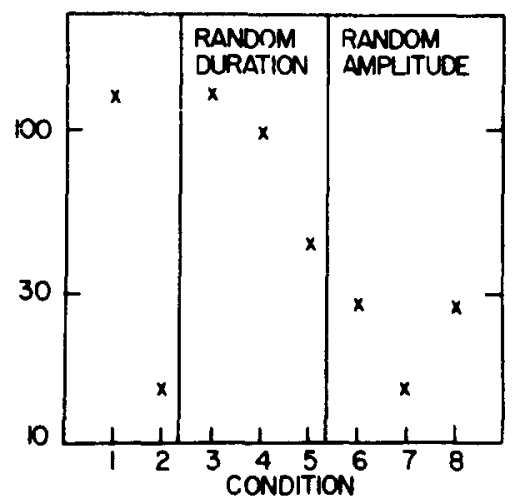

Figure 2. The just discriminable difference in gap duration for $T=100$ msec. The conditions specified on the abscissa are described in Table 1.

are also displayed in Figure 2. When the amplitudes are randomized, the change in $\Delta T$ is small, but it can be seen that it is exclusively the amplitude of the second burst that handicaps discrimination (Part 7 of Condition 6 is the same as Part 2 of Condition 6, the fixed marker). To sum up, for large $T$, the effects of randomization are due primarily to the randomization of the first marker duration. Note, however, that the lack of a large effect of marker amplitude in the random condition could possibly be due to the restricted range over which the marker amplitude varied.

\section{DISCUSSION AND CONCLUSION}

The results of these experiments clearly demonstrate that randomizing the marker duration influences the just discriminable gap duration, $\Delta T$. The question is why $\Delta T$ is so definitely affected. In the next section, we present a brief heuristic discussion of a possible mechanism for the change in $\Delta \mathrm{T}$.

\section{Two-Cue Hypothesis}

Suppose that detection in the presence of the fixed marker relies on a maximally efficient set $f$ of cues; and that similarly, detection in the presence of the random marker relies on a less efficient set $r$ of cues. If the random marker makes it impossible to utilize set $f$ of cues, then the random marker may be expected to result in larger values of $\Delta T$.

The notion here is that some cue, available in the fixed marker condition, is eliminated when the markers are randomized. The nature of the two sets of cues in random and fixed marker conditions remains uncertain, although the data indicate that whatever cue is used in the fixed marker condition is disrupted by randomizing the duration of the first marker. On a global level, the data suggest that uncertainty with respect to the first marker's duration or a changed percept due to randomization 
of the first marker handicaps performance. As Woodrow (1935) showed, the labeling of a gap is affected by the durations of the surrounding markers. But why, in either case, is discrimination affected only by the duration of the first marker and not by the first marker's intensity or by the characteristics of the second marker? One obvious possibility is that the subjects discriminate using as a cue the time between the onsets of the two markers, at least for some of the marker durations used in this experiment. The notion that onset-onset times provide the cue for the subject's judgment has also been suggested by Divenyi and Danner (1975). One test of the onset-to-onset hypothesis could be found in a trial-by-trial breakdown of our data. Unfortunately, technical difficulties allowed only the averages for each block to be collected in this study.

Finally, let us consider the question of whether $\Delta T$ must be independent of the marker characteristics if a central timing mechanism is operating. Suppose that $\Delta T$ depends on the marker duration and amplitude. It is not impossible that the central timing begins at the perceived offset of the first marker and ends at the perceived onset of the second marker. If so, the perceived duration would depend on any factors which influence the offset perception of the first marker and the onset perception of the second marker. If the marker amplitude or duration influences offset or onset judgments, then a central timing mechanism might well produce results that are dependent on the marker parameters.
In brief, an independence of duration judgments and marker characteristics lends support to the notion of central timing. However, the dependence of duration judgments on marker characteristics would not preclude the operation of a central timing mechanism. Thus, in our random marker conditions, a central timing mechanism may still be operating, but may be timing different cues than in the fixed marker conditions. In particular, the form of the results suggests to us that in at least some of the conditions, it is the onset-onset rather than the offset-onset period that is being timed.

\section{REFERENCES}

AвEl. S. M. Discrimination of temporal gaps. Joumal of the Acousticul Socicty of America, 1972. 52, 519-524.

Allan. L. G., \& Kristofferson, A. B. Psychophysical theories of duration discrimination. Penception \& Psychophysics, 1974, 16, 26-34.

Divenyi, P. L.. \& Danner, W. F. Nonmonotonic discrimination functions for time intervals: Implications for VOT perception. Journal of the Acoustical Society of America, 1975, 58, S36 (A).

Plomp. R. Rate of decay of auditory sensation. Joumal of the Acoustical Society of America, 1964, 36, 277-282.

Woodrow. H. The effect of practice upon time order errors in the comparison of temporal interals. Psychological Review, 1935. 42, 127-152.

\author{
(Received for publication June 9, 1975; \\ revision received December 2,1975 . \\ accepted January 30, 1976.)
}

\section{Erratum}

PENNER, M. J. Persistence and integration: Two consequences of a sliding integrator. Perception \& Psychophysics, 1975, 18, 114-120-The section on page 119 should read as follows:

\section{Click Detection}

The decision rule for clicks involves the comparison of the maximum value of $y(t)$ from the two intervals of the forced choice task. In Penner (1975), the "maximum" value for $y(t)$ in the interval containing the click was mathematically expressed as the value of $y(t)$ just after the click occurred. However, the maximum value of $y(t)$ does not always occur just after the click. For large $T_{3}$, the maximum value of $y(t)$ occurs at time shortly after $T_{3} / 2$ in the interval containing the signal. For small $T_{3}$, the maximum may occur at time $T_{3}$ in the interval containing the signal. The following are the corrected calculations for the case of a click in noise.
We use Equation 3 to fit the data for the detection of a click in the temporal center of a noise burst lasting $T_{3} \mathrm{msec}$. The response to the masker alone, $y_{m}$, is easily computed from Equation 3 using an exponential integrator of Equation 4 with a time constant $\xi$ :

$$
w(\xi)=e^{-t / \xi} \quad t \geqslant 0 .
$$

Let $x(t)=A$ for $0 \leqslant t \leqslant T_{3}$, and then the maximum value of $y_{m}$ occurs at time $T_{3}$ and is:

$$
y_{m}=A \xi\left(1-e^{-T_{3} / \xi}\right)
$$

